\title{
Outage Performance of Cooperative Cognitive Radio Networks under Joint Constraints of Co-Channel Interference, Intercept Probability and Hardware Imperfection
}

\author{
Pham Thi Dan Ngoc ${ }^{1,2}$, Tran Trung Duy ${ }^{2, *}$, Ho Van Khuong ${ }^{1}$ \\ ${ }^{1}$ Ho Chi Minh City University of Technology, VNU-HCM, Ho Chi Minh City, Vietnam \\ ${ }^{2}$ Posts and Telecommunications Institute of Technology, Ho Chi Minh City, Vietnam
}

\section{Abstract}

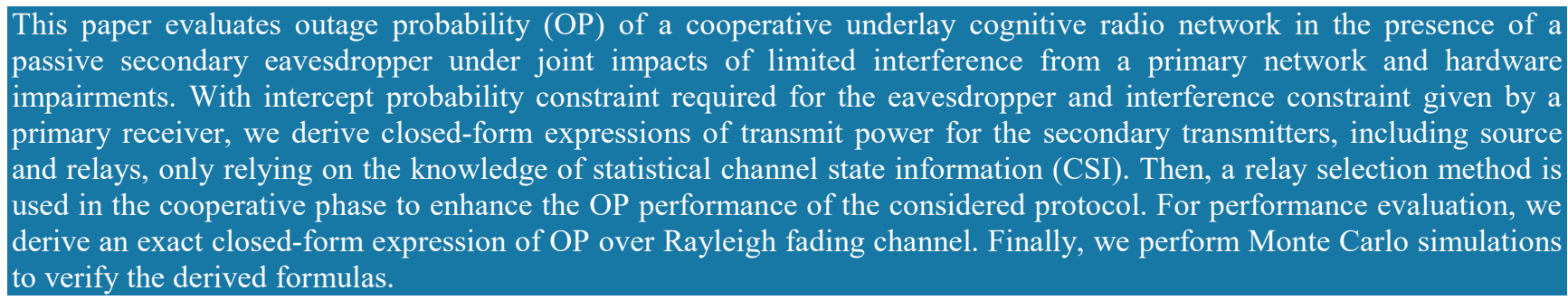

Keywords: Physical-layer security, outage probability, intercept probability, underlay cognitive radio.

Received on 04 May 2019, accepted on 22 May 2019, published on 13 June 2019

Copyright (C) 2019 Pham Thi Dan Ngoc et al., licensed to EAI. This is an open access article distributed under the terms of the Creative Commons Attribution licence (http://creativecommons.org/licenses/by/3.0/), which permits unlimited use, distribution and reproduction in any medium so long as the original work is properly cited.

doi: 10.4108/eai.13-6-2019.159124

\section{Introduction}

Recently, physical-layer security (PLS) [1]-[3] has gained much attention of researchers as a potential solution to protect the legitimate users without using complex cryptographic methods. In principle, PLS exploits physical-layer characteristics such as channel state information (CSI), link distances to obtain security for wireless communication systems. In [4]-[6], cooperative relay selection methods were proposed to improve secrecy performances in terms of average secrecy capacity, secrecy outage probability, probability of non-zero secrecy capacity. In [4]-[5], the relays are selected in the cooperative phase to protect the destination against the eavesdropper. In [6], the authors proposed a joint beam-forming and relay selection method to obtain PLS for dual-hop relaying networks. To further enhance secrecy performance, cooperative jamming (CJ)

*Corresponding author. Email: Trantrungduy@ptithcm.edu.vn techniques [7]-[9] can be used, where jammers are employed to transmit artificial noises on the eavesdropper. In [7], the authors proposed joint relay and jammer selection methods, where the selected relay is used to forward the source data to the destination, while the chosen jammer generates interference on the eavesdropper. The published work [8] evaluated secrecy performance of cooperative cognitive networks with various relay and jammer selection schemes in underlay spectrum sharing approach. In [9], the authors proposed a harvest-to-jam method, where the jammer harvests energy from radio frequency signals for generating noises. However, the implementation of the CJ methods is very complex due to high synchronization between the nodes [10]. Different with [4]-[9], the authors of [10]-[13] evaluated performance of secure communication protocols via intercept probability (IP) of the eavesdropping links and outage probability (OP) of the data links. As showed in [10]-[13], there exists a trade-off 
between security and reliability which can be improved by using relay selection methods.

To obtain high secrecy performance, CSI estimation of the eavesdropping channels has an important role. Indeed, references [14]-[17] assumed that the eavesdroppers are active nodes and hence, the legitimate transmitters/receivers can perfectly obtain the eavesdropping CSIs to optimize the secrecy capacity. However, when the eavesdroppers are passive, the protocols proposed in [14]-[17] cannot be completely applied. In [10], [18]-[19], the authors considered passive eavesdropper schemes, in which the secure transmission is enhanced by knowledge of statistical CSIs of the eavesdropping links. Particularly, the reference [10] proposed two power allocation strategies to reduce the IP value below a desired threshold, while the best relay selection using max-min strategy is used to enhance the end-to-end OP. In [18], the authors proposed a joint relay selection and power allocation method to enhance secrecy performance of a cooperative relaying network in the presence of untrusted relays and passive eavesdroppers. The authors of [19] designed a CJ method for secrecy enhancement in MIMO systems with multiple passive eavesdroppers.

In this paper, we evaluate outage performance of a secure cooperative cognitive radio protocol operating on the underlay approach [8], [13], in terms of OP. In the considered scheme, a secondary source communicates with a secondary destination via the assistance of secondary decode-and-forward (DF) relays, in presence of a passive secondary eavesdropper. Assume that only statistical CSIs of the interference and eavesdropping links are available, we propose a simple power allocation strategy for the secondary source and relays to satisfy both the interference constraint and the IP constraint. Then, we propose a relay selection method to enhance the OP performance for the secondary networks under impact of the co-channel interference from the primary network and the hardware impairments [20]-[22]. For performance evaluation, we derive exact closed-form expression of OP over Rayleigh fading channel. Finally, we perform Monte Carlo simulations to verify the theoretical results. The results presented that the transmit power of the primary transmitter, the number of relays and the position of the relays significantly impact the outage probability.

The rest of this paper is organized as follows. The system model of the proposed protocol is described in Section 2. In Section 3, the exact closed-form expression of OP is derived. The simulation results are shown in Section 4. Finally, this paper is concluded in Section 5.

\section{System Model}

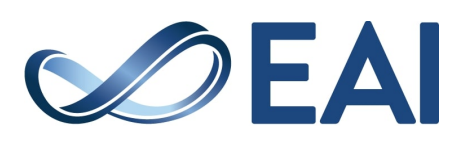

where $\lambda_{\mathrm{X}, \mathrm{Y}}=d_{\mathrm{X}, \mathrm{Y}}^{\beta}$, and $\beta$ is path-loss exponent [23][24]. Assume that the secondary relays are close together, which form a cluster, and hence $d_{\mathrm{X}, \mathrm{R}_{m}}=d_{\mathrm{X}, \mathrm{R}}$, $d_{\mathrm{R}_{m}, \mathrm{Y}}=d_{\mathrm{R}, \mathrm{Y}}$ or $\lambda_{\mathrm{X}, \mathrm{R}_{m}}=\lambda_{\mathrm{X}, \mathrm{R}}, \lambda_{\mathrm{R}_{m}, \mathrm{Y}}=\lambda_{\mathrm{R}, \mathrm{Y}}$ for all $m$.

\subsection{Operation of the proposed protocol}

At the first time slot, the source (S) broadcasts its data to the destination and all of the relays. Next, the relays attempt to decode the source data from the received signal. Let us denote $Z_{1}$ and $Z_{2}$ as the set of the relays that decode the data correctly and incorrectly, respectively. Without loss of generality, we can assume that $Z_{1}=\left\{\mathrm{R}_{1}, \mathrm{R}_{2}, \ldots, \mathrm{R}_{K}\right\}$ and $Z_{2}=\left\{\mathrm{R}_{K+1}, \mathrm{R}_{K+2}, \ldots, \mathrm{R}_{M}\right\}$, 
where $K$ is the number of the successful relays, $0 \leq K \leq M$. For example, if $K=0$, there is no relay which can retransmit the source data to the destination. If $K \geq 1$, one of the successful relays is selected by the following method:

$$
\mathrm{R}_{b}: \gamma_{\mathrm{R}_{b}, \mathrm{D}}=\max _{k=1,2, \ldots, K}\left(\gamma_{\mathrm{R}_{k}, \mathrm{D}}\right) .
$$

Equation (2) implies that the relay providing the highest channel gain to the destination is chosen for the cooperation. Indeed, the $\mathrm{R}_{b}$ relay will re-encode, and transmit the encoded data to the destination at the second time slot. Finally, the destination attempts to decode the source data from the signals received from the source and the selected relay. It is also noted that if $K=0$, the destination only uses the signal from the source for the decoding process.

Furthermore, $\mathrm{S}$ and $\mathrm{R}_{b}$ can employ the randomize-andforward (RF) technique [25]-[26] to confuse the eavesdropper. Particularly, random code-books are generated by $\mathrm{S}$ and $\mathrm{R}_{b}$ to avoid $\mathrm{E}$ to combine the received signals with maximal ratio combining (MRC).

\subsection{Interference Constraint}

The instantaneous signal-to-interference-plus-noise ratios (SINRs) obtained at PR at the first and second time slot of the secondary data transmission can be expressed, respectively as

$$
\begin{aligned}
& \psi_{\mathrm{PT}, \mathrm{PR}}^{(1)}=\frac{P_{\mathrm{PT}} \gamma_{\mathrm{PT}, \mathrm{PR}}}{\kappa_{\mathrm{P}}^{2} P_{\mathrm{PT}} \gamma_{\mathrm{PT}, \mathrm{PR}}+P_{\mathrm{S}} \gamma_{\mathrm{S}, \mathrm{PR}}+\sigma^{2}}, \\
& \psi_{\mathrm{PT}, \mathrm{PR}}^{(2)}=\frac{P_{\mathrm{PT}} \gamma_{\mathrm{PT}, \mathrm{PR}}}{\kappa_{\mathrm{P}}^{2} P_{\mathrm{PT}} \gamma_{\mathrm{PT}, \mathrm{PR}}+P_{\mathrm{R}_{b}} \gamma_{\mathrm{R}_{b}, \mathrm{PR}}+\sigma^{2}},
\end{aligned}
$$

where $\kappa_{\mathrm{P}}^{2}$ is the total level of hardware impairments of the $\mathrm{PT} \rightarrow \mathrm{PR}$ link [20]-[22], $P_{\mathrm{PT}}, P_{\mathrm{S}}, P_{\mathrm{R}_{b}}$ are transmit powers of $\mathrm{PT}, \mathrm{S}$ and $\mathrm{R}_{b}$, respectively, and $\sigma^{2}$ is variance of additive white Gaussian noise (AWGN).

Then, the channel capacities between PT and PR in the first and the second time slot can be given, respectively as

$$
\begin{aligned}
& C_{\mathrm{PT}, \mathrm{PR}}^{(1)}=\frac{1}{2} \log _{2}\left(1+\psi_{\mathrm{PT}, \mathrm{PR}}^{(1)}\right), \\
& C_{\mathrm{PT}, \mathrm{PR}}^{(2)}=\frac{1}{2} \log _{2}\left(1+\psi_{\mathrm{PT}, \mathrm{PR}}^{(2)}\right),
\end{aligned}
$$

where the factor $1 / 2$ indicates that the data transmission of the secondary network is split into two orthogonal time slots.

To protect quality of service (QoS) of the primary network at any time slots, we have to focus on the minimum value of $C_{\mathrm{PT}, \mathrm{PR}}^{(1)}$ and $C_{\mathrm{PT}, \mathrm{PR}}^{(2)}$, i.e.,

$$
\begin{aligned}
C_{\mathrm{PT}, \mathrm{PR}}^{\min } & =\min \left(C_{\mathrm{PT}, \mathrm{PR}}^{(1)}, C_{\mathrm{PT}, \mathrm{PR}}^{(2)}\right) \\
& =\frac{1}{2} \log _{2}\left[1+\min \left(\psi_{\mathrm{PT}, \mathrm{PR}}^{(1)}, \psi_{\mathrm{PT}, \mathrm{PR}}^{(2)}\right)\right] .
\end{aligned}
$$

Then, the outage probability of the primary network can be formulated as

$$
\begin{aligned}
\mathrm{OP}_{\mathrm{P}} & =\operatorname{Pr}\left(C_{\mathrm{PT}, \mathrm{PR}}^{\min }<\mathrm{C}_{\mathrm{P}}\right) \\
& =\operatorname{Pr}\left[\min \left(\psi_{\mathrm{PT}, \mathrm{PR}}^{(1)}, \psi_{\mathrm{PT}, \mathrm{PR}}^{(2)}\right)<\rho_{\mathrm{P}}\right] \\
& =1-\operatorname{Pr}\left(\psi_{\mathrm{PT}, \mathrm{PR}}^{(1)} \geq \rho_{\mathrm{P}}, \psi_{\mathrm{PT}, \mathrm{PR}}^{(2)} \geq \rho_{\mathrm{P}}\right),
\end{aligned}
$$

where $C_{P}$ is target rate of the primary network, and

$$
\rho_{\mathrm{P}}=2^{\wedge}\left(2 \mathrm{C}_{\mathrm{P}}\right)-1 \text {. }
$$

Combining (3)-(6), we can rewrite $\mathrm{OP}_{\mathrm{P}}$ under the following form:

$$
\begin{aligned}
\mathrm{OP}_{\mathrm{P}} & =1-\operatorname{Pr}\left(\gamma_{\mathrm{PT}, \mathrm{PR}} \geq \frac{\theta_{\mathrm{P}} P_{\mathrm{S}}}{P_{\mathrm{PT}}} \gamma_{\mathrm{S}, \mathrm{PR}}+\frac{\theta_{\mathrm{P}} \sigma^{2}}{P_{\mathrm{PT}}},\right. \\
& \left.\gamma_{\mathrm{PT}, \mathrm{PR}} \geq \frac{\theta_{\mathrm{P}} P_{\mathrm{R}_{b}}}{P_{\mathrm{PT}}} \gamma_{\mathrm{R}_{b}, \mathrm{PR}}+\frac{\theta_{\mathrm{P}} \sigma^{2}}{P_{\mathrm{PT}}}\right) \\
& =1-\operatorname{Pr}\left[\gamma_{\mathrm{PT}, \mathrm{PR}} \geq \max \left(Z_{1}, Z_{2}\right)\right] .
\end{aligned}
$$

where

$$
\begin{aligned}
& \theta_{\mathrm{P}}=\frac{\rho_{\mathrm{P}}}{1-\kappa_{\mathrm{P}}^{2} \rho_{\mathrm{P}}}, Z_{1}=\frac{\theta_{\mathrm{P}} P_{\mathrm{S}}}{P_{\mathrm{PT}}} \gamma_{\mathrm{S}, \mathrm{PR}}+\frac{\theta_{\mathrm{P}} \sigma^{2}}{P_{\mathrm{PT}}}, \\
& Z_{2}=\frac{\theta_{\mathrm{P}} P_{\mathrm{R}_{b}}}{P_{\mathrm{PT}}} \gamma_{\mathrm{R}_{b}, \mathrm{PR}}+\frac{\theta_{\mathrm{P}} \sigma^{2}}{P_{\mathrm{PT}}} .
\end{aligned}
$$

Denoting $Z_{\max }=\max \left(Z_{1}, Z_{2}\right)$, its $\mathrm{CDF}$ can be formulated as

$$
\begin{aligned}
F_{Z_{\text {max }}}(z) & =\operatorname{Pr}\left(Z_{1}<z\right) \operatorname{Pr}\left(Z_{2}<z\right) \\
= & \operatorname{Pr}\left(\gamma_{\mathrm{S}, \mathrm{PR}}<\frac{P_{\mathrm{PT}}}{\theta_{\mathrm{P}} P_{\mathrm{S}}} z-\frac{\sigma^{2}}{P_{\mathrm{S}}}\right) \\
& \times \operatorname{Pr}\left(\gamma_{\mathrm{R}_{b}, \mathrm{PR}}<\frac{P_{\mathrm{PT}}}{\theta_{\mathrm{P}} P_{\mathrm{R}_{b}}} z-\frac{\sigma^{2}}{P_{\mathrm{R}_{b}}}\right) .
\end{aligned}
$$

We can observe from (10) that if $z \leq \sigma^{2} \theta_{\mathrm{P}} / P_{\mathrm{PT}}$, then $F_{Z_{\max }}(z)=0$, and if $z>\sigma^{2} \theta_{\mathrm{P}} / P_{\mathrm{PT}}$, we have

$$
\begin{aligned}
F_{Z_{\max }}(z) & =\left[1-\exp \left(\frac{\lambda_{\mathrm{S}, \mathrm{PR}} \sigma^{2}}{P_{\mathrm{S}}}\right) \exp \left(-\frac{\lambda_{\mathrm{S}, \mathrm{PR}} P_{\mathrm{PT}}}{\theta_{\mathrm{P}} P_{\mathrm{S}}} z\right)\right] \\
\times & {\left[1-\exp \left(\frac{\lambda_{\mathrm{R}, \mathrm{PR}} \sigma^{2}}{P_{\mathrm{R}_{b}}}\right) \exp \left(-\frac{\lambda_{\mathrm{R}, \mathrm{PR}} P_{\mathrm{PT}}}{\theta_{\mathrm{P}} P_{\mathrm{R}_{b}}} z\right)\right] . }
\end{aligned}
$$

Therefore, if $z \leq \sigma^{2} \theta_{\mathrm{P}} / P_{\mathrm{PT}}$, then $f_{Z_{\max }}(z)=0$, and if $z>\sigma^{2} \theta_{\mathrm{P}} / P_{\mathrm{PT}}$, we can obtain PDF of $Z_{\max }$ as 


$$
\begin{aligned}
& f_{Z_{\max }}(z)=\frac{\lambda_{\mathrm{S}, \mathrm{PR}} P_{\mathrm{PT}}}{\theta_{\mathrm{P}} P_{\mathrm{S}}} \exp \left(\frac{\lambda_{\mathrm{S}, \mathrm{PR}} \sigma^{2}}{P_{\mathrm{S}}}\right) \exp \left(-\frac{\lambda_{\mathrm{S}, \mathrm{PR}} P_{\mathrm{PT}}}{\theta_{\mathrm{P}} P_{\mathrm{S}}} z\right) \\
& +\frac{\lambda_{\mathrm{R}, \mathrm{PR}} P_{\mathrm{PT}}}{\theta_{\mathrm{P}} P_{\mathrm{R}_{b}}} \exp \left(\frac{\lambda_{\mathrm{R}, \mathrm{PR}} \sigma^{2}}{P_{\mathrm{R}_{b}}}\right) \exp \left(-\frac{\lambda_{\mathrm{R}, \mathrm{PR}} P_{\mathrm{PT}}}{\theta_{\mathrm{P}} P_{\mathrm{R}_{b}}} z\right) \\
& -\left(\frac{\lambda_{\mathrm{S}, \mathrm{PR}}}{P_{\mathrm{S}}}+\frac{\lambda_{\mathrm{R}, \mathrm{PR}}}{P_{\mathrm{R}_{b}}}\right) \frac{P_{\mathrm{PT}}}{\theta_{\mathrm{P}}} \exp \left(\frac{\lambda_{\mathrm{S}, \mathrm{PR}} \sigma^{2}}{P_{\mathrm{S}}}+\frac{\lambda_{\mathrm{R}, \mathrm{PR}} \sigma^{2}}{P_{\mathrm{R}_{b}}}\right) \\
& \times \exp \left[-\left(\frac{\lambda_{\mathrm{S} \mathrm{PR}}}{P_{\mathrm{S}}}+\frac{\lambda_{\mathrm{R}, \mathrm{PR}}}{P_{\mathrm{R}_{b}}}\right) \frac{P_{\mathrm{PT}}}{\theta_{\mathrm{P}}} z\right] .
\end{aligned}
$$

Then, we can rewrite (8) as follows:

$$
\mathrm{OP}_{\mathrm{P}}=1-\int_{\frac{\sigma^{2} \theta_{\mathrm{P}}}{P_{\mathrm{PT}}}}^{+\infty}\left[1-F_{\gamma_{\mathrm{PT}, \mathrm{PR}}}(z)\right] f_{Z_{\max }}(z) d z .
$$

Substituting (1) and (12) into (13), after some manipulations, we obtain

$$
\begin{aligned}
\mathrm{OP}_{\mathrm{P}} & =1-\frac{\lambda_{\mathrm{S}, \mathrm{PR}} P_{\mathrm{PT}}}{\lambda_{\mathrm{PT}, \mathrm{PR}} \theta_{\mathrm{P}} P_{\mathrm{S}}+\lambda_{\mathrm{S}, \mathrm{PR}} P_{\mathrm{PT}}} \exp \left(-\frac{\lambda_{\mathrm{PT}, \mathrm{PR}} \theta_{\mathrm{P}} \sigma^{2}}{P_{\mathrm{PT}}}\right) \\
& -\frac{\lambda_{\mathrm{R}, \mathrm{PR}} P_{\mathrm{PT}}}{\lambda_{\mathrm{PT}, \mathrm{PR}} \theta_{\mathrm{P}} P_{\mathrm{R}_{b}}+\lambda_{\mathrm{R}, \mathrm{PR}} P_{\mathrm{PT}}} \exp \left(-\frac{\lambda_{\mathrm{PT}, \mathrm{PR}} \theta_{\mathrm{P}} \sigma^{2}}{P_{\mathrm{PT}}}\right) \\
& +\left(\frac{\lambda_{\mathrm{S}, \mathrm{PR}}}{P_{\mathrm{S}}}+\frac{\lambda_{\mathrm{R}, \mathrm{PR}}}{P_{\mathrm{R}_{b}}}\right) \exp \left(-\frac{\lambda_{\mathrm{PT}, \mathrm{PR}} \theta_{\mathrm{P}} \sigma^{2}}{P_{\mathrm{PT}}}\right) \times \\
& \frac{P_{\mathrm{PT}}}{\lambda_{\mathrm{PT}, \mathrm{PR}} \theta_{\mathrm{P}}+\left(\lambda_{\mathrm{S}, \mathrm{PR}} / P_{\mathrm{S}}+\lambda_{\mathrm{R}, \mathrm{PR}} / P_{\mathrm{R}_{b}}\right) P_{\mathrm{PT}}} .
\end{aligned}
$$

Next, we propose a simple power allocation for the secondary transmitters to satisfy the QoS of the primary network, i.e., $\mathrm{OP}_{\mathrm{P}} \leq \varepsilon_{\mathrm{OP}}$, where $\varepsilon_{\mathrm{OP}}$ is a pre-determined OP value. Now, we observe that if the S-PR distance is longer than the $\mathrm{R}_{b}-\mathrm{PR}$ distance, then $P_{\mathrm{S}}$ should be higher than $P_{\mathrm{R}_{b}}$, and vice versa. Hence, we have

$$
\frac{P_{\mathrm{S}}}{\lambda_{\mathrm{S}, \mathrm{PR}}}=\frac{P_{\mathrm{R}_{b}}}{\lambda_{\mathrm{R}, \mathrm{PR}}}=\chi_{1} .
$$

Substituting (15) into (14), which yields

$$
\mathrm{OP}_{\mathrm{P}}=1-\frac{2 \mu}{\left(\alpha_{1} \chi_{1}+1\right)\left(\alpha_{1} \chi_{1}+2\right)},
$$

where

$$
\alpha_{1}=\frac{\lambda_{\mathrm{PT}, \mathrm{PR}} \theta_{\mathrm{P}}}{P_{\mathrm{PT}}}, \mu=\exp \left(-\frac{\lambda_{\mathrm{PT}, \mathrm{PR}} \theta_{\mathrm{P}} \sigma^{2}}{P_{\mathrm{PT}}}\right) .
$$

Solving $\mathrm{OP}_{\mathrm{P}}=\varepsilon_{\mathrm{OP}}$, we can obtain

$$
\chi_{1}=\frac{-3 \pm \sqrt{9-4 \vartheta}}{2 \alpha_{1}},
$$

where $\vartheta=2-2 \mu /\left(1-\varepsilon_{\mathrm{OP}}\right)$, and $9 \geq 4 \vartheta$.

Because the transmit power $P_{\mathrm{S}}$ and $P_{\mathrm{R}_{b}}$ are not negative, we have

$$
\begin{aligned}
& P_{\mathrm{S}} \leq P_{\mathrm{S}}^{*}=\left[\left(\frac{-3+\sqrt{9-4 \vartheta}}{2 \alpha_{1}}\right) \lambda_{\mathrm{S}, \mathrm{PR}}\right]^{+}, \\
& P_{\mathrm{R}_{b}} \leq P_{\mathrm{R}_{b}}^{*}=\left[\left(\frac{-3+\sqrt{9-4 \vartheta}}{2 \alpha_{1}}\right) \lambda_{\mathrm{R}, \mathrm{PR}}\right]^{+},
\end{aligned}
$$

where $[x]^{+}=\max (0, x)$.

\subsection{IP Constraint}

Similar to (4), the instantaneous channel capacity obtained at the eavesdropper (E) at the first and second time slot can be expressed, respectively as

$$
\begin{aligned}
& C_{\mathrm{S}, \mathrm{E}}=\frac{1}{2} \log _{2}\left(1+\frac{P_{\mathrm{S}} \gamma_{\mathrm{S}, \mathrm{E}}}{\kappa_{\mathrm{E}}^{2} P_{\mathrm{S}} \gamma_{\mathrm{S}, \mathrm{E}}+P_{\mathrm{PT}} \gamma_{\mathrm{PT}, \mathrm{E}}+\sigma^{2}}\right), \\
& C_{\mathrm{R}_{b}, \mathrm{E}}=\frac{1}{2} \log _{2}\left(1+\frac{P_{\mathrm{R}_{b}} \gamma_{\mathrm{R}_{b}, \mathrm{E}}}{\kappa_{\mathrm{E}}^{2} P_{\mathrm{R}_{b}} \gamma_{\mathrm{R}_{b}, \mathrm{E}}+P_{\mathrm{PT}} \gamma_{\mathrm{PT}, \mathrm{E}}+\sigma^{2}}\right),
\end{aligned}
$$

where $\kappa_{\mathrm{E}}^{2}$ is the total level of hardware impairments of all the eavesdropping links, i.e., $\mathrm{S} \rightarrow \mathrm{E}, \mathrm{R}_{b} \rightarrow \mathrm{E}$. Moreover, when $P_{\mathrm{PT}} ? \sigma^{2}$, we can approximate (20) as

$$
\begin{aligned}
& C_{\mathrm{S}, \mathrm{E}} \approx \frac{1}{2} \log _{2}\left(1+\frac{P_{\mathrm{S}} \gamma_{\mathrm{S}, \mathrm{E}}}{\kappa_{\mathrm{E}}^{2} P_{\mathrm{S}} \gamma_{\mathrm{S}, \mathrm{E}}+P_{\mathrm{PT}} \gamma_{\mathrm{PT}, \mathrm{E}}}\right), \\
& C_{\mathrm{R}_{b}, \mathrm{E}} \approx \frac{1}{2} \log _{2}\left(1+\frac{P_{\mathrm{R}_{b}} \gamma_{\mathrm{R}_{b}, \mathrm{E}}}{\kappa_{\mathrm{E}}^{2} P_{\mathrm{R}_{b}} \gamma_{\mathrm{R}_{b}, \mathrm{E}}+P_{\mathrm{PT}} \gamma_{\mathrm{PT}, \mathrm{E}}}\right),
\end{aligned}
$$

Moreover, because the RF technique is employed by $\mathrm{S}$ and $\mathrm{R}_{b}$, the intercept probability (IP) can be formulated, similar to [10] as

$$
\mathrm{IP}=\operatorname{Pr}\left[\max \left(C_{\mathrm{S}, \mathrm{E}}, C_{\mathrm{R}_{b}, \mathrm{E}}\right) \geq C_{\mathrm{S}}\right],
$$

where $C_{\mathrm{S}}$ is a target rate of the secondary network. Substituting (21) into (22), we obtain (23) as

$$
\begin{aligned}
\mathrm{IP} & \approx 1-\operatorname{Pr}\left(\frac{P_{\mathrm{S}} \gamma_{\mathrm{S}, \mathrm{E}}}{\kappa_{\mathrm{E}}^{2} P_{\mathrm{S}} \gamma_{\mathrm{S}, \mathrm{E}}+P_{\mathrm{PT}} \gamma_{\mathrm{PT}, \mathrm{E}}}<\rho_{\mathrm{S}},\right. \\
& \left.\frac{P_{\mathrm{R}_{b}} \gamma_{\mathrm{R}_{b}, \mathrm{E}}}{\kappa_{\mathrm{E}}^{2} P_{\mathrm{R}_{b}} \gamma_{\mathrm{R}_{b}, \mathrm{E}}+P_{\mathrm{PT}} \gamma_{\mathrm{PT}, \mathrm{E}}}<\rho_{\mathrm{S}}\right) \\
& \approx 1-\operatorname{Pr}\left(\gamma_{\mathrm{S}, \mathrm{E}}<\frac{P_{\mathrm{PT}} \theta_{\mathrm{E}}}{P_{\mathrm{S}}} \gamma_{\mathrm{PT}, \mathrm{E}}, \gamma_{\mathrm{R}_{b}, \mathrm{E}}<\frac{P_{\mathrm{PT}} \theta_{\mathrm{E}}}{P_{\mathrm{R}_{b}}} \gamma_{\mathrm{PT}, \mathrm{E}}\right) .
\end{aligned}
$$

where

$$
\rho_{\mathrm{S}}=2^{\wedge}\left(2 \mathrm{C}_{\mathrm{S}}\right)-1, \theta_{\mathrm{E}}=\frac{\rho_{\mathrm{S}}}{1-\kappa_{\mathrm{E}}^{2} \rho_{\mathrm{S}}} .
$$

It is noted from (23) that IP $=0$ if $1-\kappa_{\mathrm{E}}^{2} \rho_{\mathrm{S}} \leq 0$. Hence, in the following, we only consider the case of $1-\kappa_{\mathrm{E}}^{2} \rho_{\mathrm{S}}>0$. Now, setting $\gamma_{\mathrm{PT}, \mathrm{E}}=x$, IP conditioned on $x$ is given as 


$$
\begin{aligned}
\operatorname{IP}(x) & \approx 1-F_{\gamma_{\mathrm{S}, \mathrm{E}}}\left(\frac{P_{\mathrm{PT}} \theta_{\mathrm{E}}}{P_{\mathrm{S}}} x\right) F_{\gamma_{\mathrm{R}}, \mathrm{E}}\left(\frac{P_{\mathrm{PT}} \theta_{\mathrm{E}}}{P_{\mathrm{R}_{b}}} x\right) \\
& \approx \exp \left(-\frac{\lambda_{\mathrm{S}, \mathrm{E}} P_{\mathrm{PT}} \theta_{\mathrm{E}}}{P_{\mathrm{S}}} x\right)+\exp \left(-\frac{\lambda_{\mathrm{R}, \mathrm{E}} P_{\mathrm{PT}} \theta_{\mathrm{E}}}{P_{\mathrm{R}_{b}}} x\right) \\
& -\exp \left[-\left(\frac{\lambda_{\mathrm{S}, \mathrm{E}}}{P_{\mathrm{S}}}+\frac{\lambda_{\mathrm{R}, \mathrm{E}}}{P_{\mathrm{R}_{b}}}\right) P_{\mathrm{PT}} \theta_{\mathrm{E}} x\right] .
\end{aligned}
$$

Then, IP can be approximated by

$$
\begin{aligned}
\mathrm{IP} & =\int_{0}^{+\infty} \mathrm{IP}(x) f_{\gamma_{\mathrm{PT}, \mathrm{E}}}(x) d x \\
& \approx \frac{\lambda_{\mathrm{PT}, \mathrm{E}}}{\lambda_{\mathrm{PT}, \mathrm{E}}+\lambda_{\mathrm{S}, \mathrm{E}} P_{\mathrm{PT}} \theta_{\mathrm{E}} / P_{\mathrm{S}}}+\frac{\lambda_{\mathrm{PT}, \mathrm{E}}}{\lambda_{\mathrm{PT}, \mathrm{E}}+\lambda_{\mathrm{R}, \mathrm{E}} P_{\mathrm{PT}} \theta_{\mathrm{E}} / P_{\mathrm{R}_{b}}} \\
& -\frac{\lambda_{\mathrm{PT}, \mathrm{E}}}{\lambda_{\mathrm{PT}, \mathrm{E}}+\left(\lambda_{\mathrm{S}, \mathrm{E}} / P_{\mathrm{S}}+\lambda_{\mathrm{R}, \mathrm{E}} / P_{\mathrm{R}_{b}}\right) P_{\mathrm{PT}} \theta_{\mathrm{E}}} .
\end{aligned}
$$

Similar to [10], IP must be below a designed value $\varepsilon_{\mathrm{IP}}$, i.e., $\mathrm{IP} \leq \varepsilon_{\mathrm{IP}}$. Moreover, similar to (15), we propose a simple power allocation strategy as

$$
\frac{P_{\mathrm{S}}}{\lambda_{\mathrm{S}, \mathrm{E}}}=\frac{P_{\mathrm{R}_{b}}}{\lambda_{\mathrm{R}, \mathrm{E}}}=\chi_{2}
$$

Substituting (27) into (26), we obtain (28) as

$$
\mathrm{IP} \approx \frac{\chi_{2}\left(\chi_{2}+3 \alpha_{2}\right)}{\left(\chi_{2}+\alpha_{2}\right)\left(\chi_{2}+2 \alpha_{2}\right)}
$$

where $\alpha_{2}=P_{\mathrm{PT}} \theta_{\mathrm{E}} / \lambda_{\mathrm{PT}, \mathrm{E}}$.

Using (28) to solve equation $\mathrm{IP}=\varepsilon_{\mathrm{IP}}$, we have

$$
\chi_{2}=\sqrt{\frac{9-\varepsilon_{\mathrm{IP}}}{1-\varepsilon_{\mathrm{IP}}}} \frac{\alpha_{2}}{2}-\frac{3 \alpha_{2}}{2} .
$$

From (29), the transmit powers of the source and the selected relay are constrained as

$$
\begin{aligned}
& P_{\mathrm{S}} \leq P_{\mathrm{S}}^{* *}=\left(\sqrt{\frac{9-\varepsilon_{\mathrm{IP}}}{1-\varepsilon_{\mathrm{IP}}}} \frac{\alpha_{2}}{2}-\frac{3 \alpha_{2}}{2}\right) \lambda_{\mathrm{S}, \mathrm{E}}, \\
& P_{\mathrm{R}_{b}} \leq P_{\mathrm{R}_{b}}^{* *}=\left(\sqrt{\frac{9-\varepsilon_{\mathrm{IP}}}{1-\varepsilon_{\mathrm{IP}}}} \frac{\alpha_{2}}{2}-\frac{3 \alpha_{2}}{2}\right) \lambda_{\mathrm{R}, \mathrm{E}} .
\end{aligned}
$$

\subsection{Transmit Power and OP Formulation}

From (19) and (30), we can give an exact closed-form expression of the transmit power for the source $(\mathrm{S})$ and the selected relay $\left(\mathrm{R}_{b}\right)$ as

$$
P_{\mathrm{S}}=\min \left(P_{\mathrm{S}}^{*}, P_{\mathrm{S}}^{* *}\right), P_{\mathrm{R}_{b}}=\min \left(P_{\mathrm{R}_{b}}^{*}, P_{\mathrm{R}_{b}}^{* *}\right) \text {. }
$$

Next, the instantaneous channel capacity of the $\mathrm{S} \rightarrow \mathrm{D}, \mathrm{S} \rightarrow \mathrm{R}_{m}$ and $\mathrm{R}_{m} \rightarrow \mathrm{D}$ links can be expressed, respectively as

$$
C_{\mathrm{S}, \mathrm{D}}=\frac{1}{2} \log _{2}\left(1+\frac{P_{\mathrm{S}} \gamma_{\mathrm{S}, \mathrm{D}}}{\kappa_{\mathrm{D}}^{2} P_{\mathrm{S}} \gamma_{\mathrm{S}, \mathrm{D}}+P_{\mathrm{PT}} \gamma_{\mathrm{PT}, \mathrm{D}}+\sigma^{2}}\right),
$$

$$
\begin{aligned}
C_{\mathrm{S}, \mathrm{R}_{m}} & =\frac{1}{2} \log _{2}\left(1+\frac{P_{\mathrm{S}} \gamma_{\mathrm{S}, \mathrm{R}_{m}}}{\kappa_{\mathrm{D}}^{2} P_{\mathrm{S}} \gamma_{\mathrm{S}, \mathrm{R}_{m}}+P_{\mathrm{PT}} \gamma_{\mathrm{PT}, \mathrm{R}_{m}}+\sigma^{2}}\right) \\
C_{\mathrm{R}_{m}, \mathrm{D}} & =\frac{1}{2} \log _{2}\left(1+\frac{P_{\mathrm{R}_{m}} \gamma_{\mathrm{R}_{m}, \mathrm{D}}}{\kappa_{\mathrm{D}}^{2} P_{\mathrm{R}_{m}} \gamma_{\mathrm{R}_{m}, \mathrm{D}}+P_{\mathrm{PT}} \gamma_{\mathrm{PT}, \mathrm{D}}+\sigma^{2}}\right)
\end{aligned}
$$

where $\kappa_{\mathrm{D}}^{2}$ is the total level of hardware impairments of all the data links. Then, we can formulate $O P$ of the considered protocol as follows:

$$
\begin{aligned}
\mathrm{OP}_{\mathrm{S}} & =\operatorname{Pr}(K=0) \operatorname{Pr}\left(C_{\mathrm{S}, \mathrm{D}}<C_{\mathrm{S}}\right) \\
& +\sum_{k=1}^{M} C_{M}^{k} \operatorname{Pr}(K=k) \underset{1444444442^{\prime} 444444444 \mathrm{Pr}}{\operatorname{Pr}\left(C_{3},\right.}
\end{aligned}
$$

where $\operatorname{Pr}(K=k)$ is obtained by

$$
\begin{gathered}
\operatorname{Pr}(K=k)=\operatorname{Pr}\left(C_{\mathrm{S}, \mathrm{R}_{1}} \geq C_{\mathrm{S}}, \ldots, C_{\mathrm{S}_{\mathrm{R}}} \geq C_{\mathrm{S}},\right. \\
\left.C_{\mathrm{S}, \mathrm{R}_{k+1}}<C_{\mathrm{S}}, \ldots, C_{\mathrm{S}_{\mathrm{R}}}<C_{\mathrm{S}}\right) .
\end{gathered}
$$

In case that $k=0$, we have

$$
\operatorname{Pr}(K=0)=\operatorname{Pr}\left(C_{\mathrm{S}, \mathrm{R}_{1}}<C_{\mathrm{S}}, C_{\mathrm{S}, \mathrm{R}_{2}}<C_{\mathrm{S}}, \ldots, C_{\mathrm{S}, \mathrm{R}_{M}}<C_{\mathrm{S}}\right) .
$$

\section{Performance Analysis}

In this section, we derive the exact closed-form expression of $\mathrm{OP}_{\mathrm{S}}$. At first, we attempt to calculate the probability $\operatorname{Pr}(K=k)$ in (35). By combining (33) and (36), we can write

$$
\begin{aligned}
\operatorname{Pr}(K=k) & =\prod_{m=1}^{k} \operatorname{Pr}\left(\gamma_{\mathrm{S}, \mathrm{R}_{m}} \geq \omega_{1} \gamma_{\mathrm{PT}, \mathrm{R}_{m}}+\omega_{2}\right) \\
& \times \prod_{m=k+1}^{M} \operatorname{Pr}\left(\gamma_{\mathrm{S}, \mathrm{R}_{m}}<\omega_{1} \gamma_{\mathrm{PT}, \mathrm{R}_{m}}+\omega_{2}\right) .
\end{aligned}
$$

where

$$
\theta_{\mathrm{D}}=\frac{\rho_{\mathrm{S}}}{1-\kappa_{\mathrm{D}}^{2} \rho_{\mathrm{S}}}, \omega_{1}=\frac{P_{\mathrm{PT}}}{P_{\mathrm{S}}} \theta_{\mathrm{D}}, \omega_{2}=\frac{\sigma^{2} \theta_{\mathrm{D}}}{P_{\mathrm{S}}} .
$$

Furthermore, equation (38) can be expressed as

$$
\begin{aligned}
& \operatorname{Pr}(K=k) \\
& =\prod_{m=1}^{k}\left[\int_{0}^{+\infty}\left(1-F_{\gamma_{\mathrm{S}_{R},}}\left(\omega_{1} x+\omega_{2}\right)\right) f_{\gamma_{\mathrm{PT}, R_{m}}}(x) d x\right] \\
& \times \prod_{m=k+1}^{M}\left[\int_{0}^{+\infty} F_{\gamma_{\mathrm{S}_{m}, R_{m}}}\left(\omega_{1} x+\omega_{2}\right) f_{\gamma_{\mathrm{T}, R_{m}}}(x) d x\right] .
\end{aligned}
$$

Substituting (1) into (40), after some manipulations, we obtain (41) as

$$
\begin{aligned}
\operatorname{Pr}(K=k) & =\left[\frac{\lambda_{\mathrm{PT}, \mathrm{R}}}{\lambda_{\mathrm{PT}, \mathrm{R}}+\lambda_{\mathrm{S}, \mathrm{R}} \omega_{1}} \exp \left(-\lambda_{\mathrm{S}, \mathrm{R}} \omega_{2}\right)\right]^{k} \\
& \times\left[1-\frac{\lambda_{\mathrm{PT}, \mathrm{R}}}{\lambda_{\mathrm{PT}, \mathrm{R}}+\lambda_{\mathrm{S}, \mathrm{R}} \omega_{1}} \exp \left(-\lambda_{\mathrm{S}, \mathrm{R}} \omega_{2}\right)\right]^{M-k} .
\end{aligned}
$$

Then, when $K=0$, from (41), we have 


$$
\operatorname{Pr}(K=0)=\left[1-\frac{\lambda_{\mathrm{PT}, \mathrm{R}}}{\lambda_{\mathrm{PT}, \mathrm{R}}+\lambda_{\mathrm{S}, \mathrm{R}} \omega_{1}} \exp \left(-\lambda_{\mathrm{S}, \mathrm{R}} \omega_{2}\right)\right]^{M} .
$$

Similar to (38)-(41), we can calculate $\operatorname{Pr}\left(C_{\mathrm{S}, \mathrm{D}}<C_{\mathrm{S}}\right)$ in (35) exactly as

$$
\begin{aligned}
\operatorname{Pr}\left(C_{\mathrm{S}, \mathrm{D}}<C_{\mathrm{S}}\right) & =\operatorname{Pr}\left(\gamma_{\mathrm{S}, \mathrm{D}}<\omega_{1} \gamma_{\mathrm{PT}, \mathrm{D}}+\omega_{2}\right) \\
& =1-\frac{\lambda_{\mathrm{PT}, \mathrm{D}}}{\lambda_{\mathrm{PT}, \mathrm{D}}+\lambda_{\mathrm{S}, \mathrm{D}} \omega_{1}} \exp \left(-\lambda_{\mathrm{S}, \mathrm{D}} \omega_{2}\right) .
\end{aligned}
$$

Finally, the probability $I=\operatorname{Pr}\left(C_{\mathrm{S}, \mathrm{D}}<C_{\mathrm{S}}, C_{\mathrm{R}_{b}, \mathrm{D}}<C_{\mathrm{S}}\right)$ in (35) can be formulated as

$$
I=\operatorname{Pr}\left(\gamma_{\mathrm{S}, \mathrm{D}}<\omega_{1} \gamma_{\mathrm{PT}, \mathrm{D}}+\omega_{2}, \gamma_{\mathrm{R}_{b}, \mathrm{D}}<\omega_{3} \gamma_{\mathrm{PT}, \mathrm{D}}+\omega_{4}\right),
$$

where

$$
\omega_{3}=\frac{P_{\mathrm{PT}}}{P_{\mathrm{R}_{b}}} \theta_{\mathrm{D}}, \omega_{4}=\frac{\sigma^{2} \theta_{\mathrm{D}}}{P_{\mathrm{R}_{b}}} .
$$

Setting $\gamma_{\mathrm{PT}, \mathrm{D}}=x$, the probability $I$ conditioned on $x$ can be given as

$$
I(x)=F_{\gamma_{\mathrm{S}, \mathrm{D}}}\left(\omega_{1} x+\omega_{2}\right) \times F_{\gamma_{\mathrm{R}, \mathrm{D}}}\left(\omega_{3} x+\omega_{4}\right) .
$$

Since $\gamma_{\mathrm{R}_{b}, \mathrm{D}}=\max _{j=1,2, \ldots, k}\left(\gamma_{\mathrm{R}_{j}, \mathrm{D}}\right)$, the $\operatorname{CDF} F_{\gamma_{\mathrm{R}_{b}, \mathrm{D}}}\left(\omega_{3} x+\omega_{4}\right)$ can be given as

$$
\begin{aligned}
F_{\gamma_{\mathrm{R}_{b} \mathrm{D}}}\left(\omega_{3} x+\omega_{4}\right) & =\operatorname{Pr}\left(\gamma_{\mathrm{R}_{b}, \mathrm{D}}<\omega_{3} x+\omega_{4}\right) \\
& =\left[1-\exp \left(-\lambda_{\mathrm{R}, \mathrm{D}}\left(\omega_{3} x+\omega_{4}\right)\right)\right]^{k} .
\end{aligned}
$$

Combining (1), (46) and (47), we have

$$
\begin{aligned}
I(x) & =\left[1-\exp \left(-\lambda_{\mathrm{S}, \mathrm{D}} \omega_{2}\right) \exp \left(-\lambda_{\mathrm{S}, \mathrm{D}} \omega_{1} x\right)\right] \\
& \times \sum_{j=0}^{k}(-1)^{j} C_{k}^{j} \exp \left(-j \lambda_{\mathrm{R}, \mathrm{D}} \omega_{4}\right) \exp \left(-j \lambda_{\mathrm{R}, \mathrm{D}} \omega_{3} x\right) \\
& =\sum_{j=0}^{k}(-1)^{j} C_{k}^{j} \exp \left(-j \lambda_{\mathrm{R}, \mathrm{D}} \omega_{4}\right) \exp \left(-j \lambda_{\mathrm{R}, \mathrm{D}} \omega_{3} x\right) \\
& -\sum_{j=0}^{k}(-1)^{j} C_{k}^{j} \exp \left[-\left(\lambda_{\mathrm{S}, \mathrm{D}} \omega_{2}+j \lambda_{\mathrm{R}, \mathrm{D}} \omega_{4}\right)\right] \\
& \times \exp \left[-\left(\lambda_{\mathrm{S}, \mathrm{D}} \omega_{1}+j \lambda_{\mathrm{R}, \mathrm{D}} \omega_{3}\right) x\right] .
\end{aligned}
$$

From (48), the probability $I$ can be calculated as

$$
\begin{aligned}
I= & \int_{0}^{+\infty} I(x) f_{\gamma_{\mathrm{P}, \mathrm{D}}}(x) \\
= & \sum_{j=0}^{k} \frac{(-1)^{j} C_{k}^{j} \lambda_{\mathrm{PT}, \mathrm{D}}}{\lambda_{\mathrm{PT}, \mathrm{D}}+j \lambda_{\mathrm{R}, \mathrm{D}} \omega_{3}} \exp \left(-j \lambda_{\mathrm{R}, \mathrm{D}} \omega_{4}\right) \\
- & \sum_{j=0}^{k} \frac{(-1)^{j} C_{k}^{j} \lambda_{\mathrm{PT}, \mathrm{D}}}{\lambda_{\mathrm{PT}, \mathrm{D}}+\lambda_{\mathrm{S}, \mathrm{D}} \omega_{1}+j \lambda_{\mathrm{R}, \mathrm{D}} \omega_{3}} \\
& \times \exp \left[-\left(\lambda_{\mathrm{S}, \mathrm{D}} \omega_{2}+j \lambda_{\mathrm{R}, \mathrm{D}} \omega_{4}\right)\right] .
\end{aligned}
$$

Finally, substituting (41), (42), (43) and (49) into (35), we obtain an exact closed-form expression of $\mathrm{OP}_{\mathrm{S}}$.

\section{Simulation Results}

In this section, we present Monte Carlo simulations to verify the theoretical results. In a two-dimensional network, we assume that the co-ordinates of the source, the destination, the relays, the eavesdropper, the primary transmitter, the primary receiver are $(0,0),(1,0)$, $\left(x_{\mathrm{R}}, 0\right),\left(x_{\mathrm{E}}, y_{\mathrm{E}}\right),(0.5,1.5)$ and $(0.5,0.75)$ respectively. In all of the simulations, we fix the path-loss exponent $(\beta)$ by 3 , the variance of $\operatorname{AWGN}\left(\sigma^{2}\right)$ by 1 , the required QoS of the primary network $\left(\varepsilon_{\mathrm{OP}}\right)$ by 0.05 , and the target rate of the primary network $\left(C_{\mathrm{P}}\right)$ by 1 .

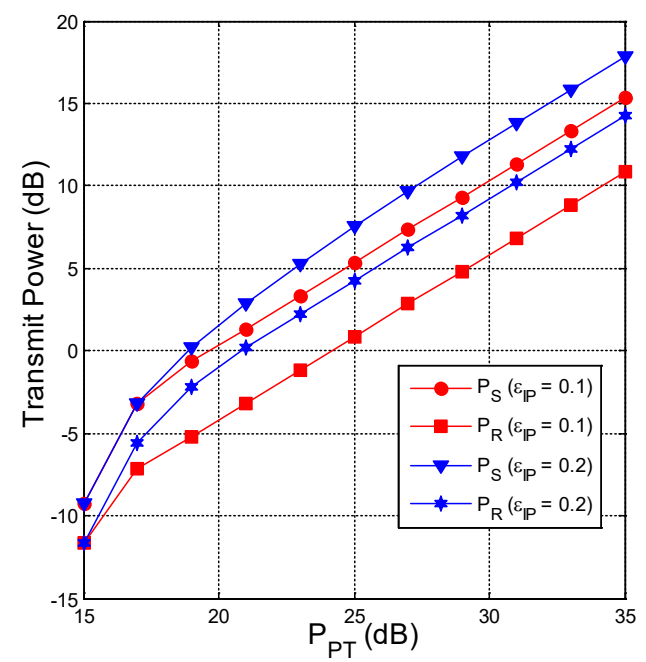

Figure 2. $\mathrm{P}_{\mathrm{S}}$ and $\mathrm{P}_{\mathrm{R}}$ as a function of $P_{\mathrm{PT}}(\mathrm{dB})$ when $x_{\mathrm{R}}=0.5, x_{\mathrm{E}}=y_{\mathrm{E}}=0.5, \kappa_{\mathrm{P}}^{2}=0.01, \kappa_{\mathrm{E}}^{2}=0.05$, and

$$
C_{\mathrm{S}}=0.25 \text {. }
$$

Figure 2 presents the transmit power of the secondary transmitters including source and relays as a function of $P_{\mathrm{PT}}$ in dB. As we can see, the transmit powers $P_{\mathrm{S}}$ and $P_{\mathrm{R}}$ increases as increasing $P_{\mathrm{PT}}$. It is due to the fact that at high transmit power $P_{\mathrm{PT}}$, the QoS of the primary network can be still satisfied with high transmit power of the secondary transmitters. It is also seen from Fig. 2 that the source and the relays can use high transmit power with higher value of $\varepsilon_{\mathrm{IP}}$.

In Fig. 3, we present the outage probability of the primary network $\left(\mathrm{OP}_{\mathrm{P}}\right)$ and the intercept probability at the eavesdropper (IP) as function of $P_{\mathrm{PT}}$ in $\mathrm{dB}$. As shown in Fig. 3, $\mathrm{OP}_{\mathrm{P}}$ and IP are below the values of $\varepsilon_{\mathrm{OP}}$ and $\varepsilon_{\mathrm{IP}}$ , respectively, which means that the proposed power allocation not only guarantees the QoS of the primary network but also reduces the intercept possibility of the eavesdropper as expected. It is worth noting that the simulation results (Sim) in Fig. 3 match very well with the theoretical ones (Analysis), which verify our derivations. 


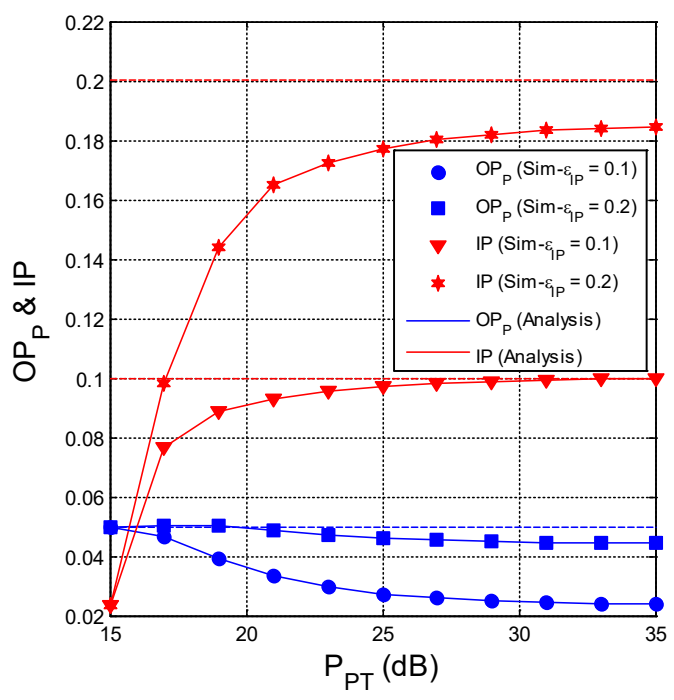

Figure 3. $\mathrm{OP}_{\mathrm{P}}$ and $\mathrm{IP}$ as a function of $P_{\mathrm{PT}}(\mathrm{dB})$ when $x_{\mathrm{R}}=0.5, x_{\mathrm{E}}=y_{\mathrm{E}}=0.5, \kappa_{\mathrm{P}}^{2}=0.01, \kappa_{\mathrm{E}}^{2}=0.05$, and

$$
C_{\mathrm{S}}=0.25 \text {. }
$$

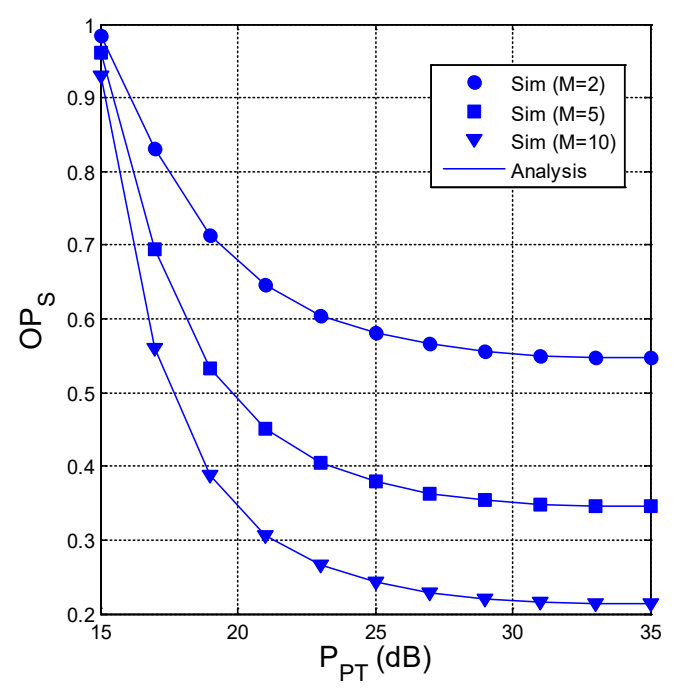

Figure 4. $\mathrm{OP}_{\mathrm{S}}$ as a function of $P_{\mathrm{PT}}(\mathrm{dB})$ when $x_{\mathrm{R}}=0.5$,

$$
\begin{gathered}
x_{\mathrm{E}}=y_{\mathrm{E}}=0.5, \kappa_{\mathrm{P}}^{2}=0.01, \kappa_{\mathrm{D}}^{2}=\kappa_{\mathrm{E}}^{2}=0.05, C_{\mathrm{S}}=0.25, \\
\text { and } \varepsilon_{\mathrm{IP}}=0.25 .
\end{gathered}
$$

In Fig. 4, we present the outage probability of the secondary network $\left(\mathrm{OP}_{\mathrm{S}}\right)$ as a function of $P_{\mathrm{PT}}$ in $\mathrm{dB}$ with different values of $M$. As seen from this figure, the outage performance of the considered protocol is much better with high number of relays. It is also observed that the $\mathrm{OP}_{\mathrm{S}}$ values decrease with the increasing of $P_{\mathrm{PT}}$. Again, the simulation results verify the theoretical ones.

Figure 5 shows $\mathrm{OP}$ of the secondary network $\left(\mathrm{OP}_{\mathrm{S}}\right)$ as a function of $\kappa_{\mathrm{D}}^{2}$ with different positions of the relays. As we can see, the outage performance is worse as the hardware impairment level increases. In addition, we also see that the value of $x_{\mathrm{R}}$ also significantly impacts on the outage performance. Indeed, the considered protocol obtains the best and worst performance as $x_{\mathrm{R}}=0.7$ and $x_{\mathrm{R}}=0.3$, respectively. Moreover, the gap between the best and worst performance is very high.

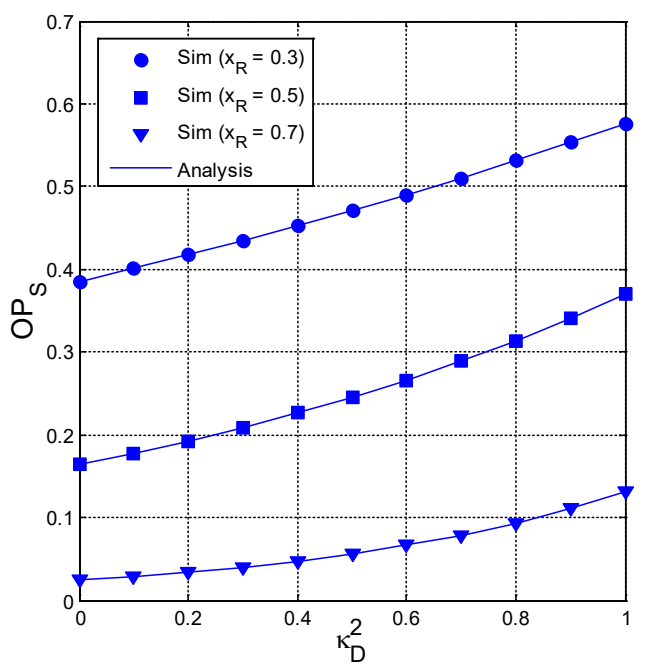

Figure 5. $\mathrm{OP}_{\mathrm{S}}$ as a function of $\kappa_{\mathrm{D}}^{2}$ when $P_{\mathrm{PT}}=25(\mathrm{~dB})$, $x_{\mathrm{E}}=y_{\mathrm{E}}=0.5, \kappa_{\mathrm{P}}^{2}=0, \kappa_{\mathrm{E}}^{2}=0.3, C_{\mathrm{S}}=0.25, M=15$, and $\varepsilon_{\mathrm{IP}}=0.25$.

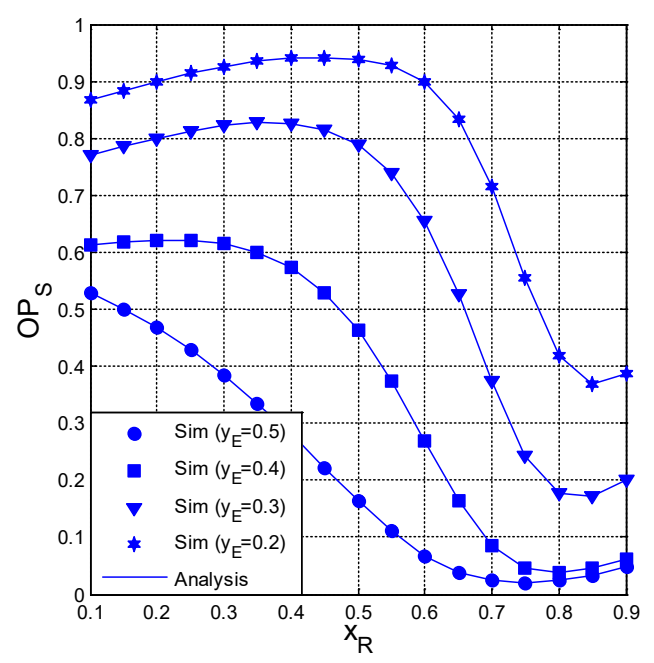

Figure 6. $\mathrm{OP}_{\mathrm{S}}$ as a function of $x_{\mathrm{R}}$ when $P_{\mathrm{PT}}=25(\mathrm{~dB})$,

$$
\begin{aligned}
x_{\mathrm{E}}=0.5, \kappa_{\mathrm{P}}^{2}=\kappa_{\mathrm{D}}^{2}=\kappa_{\mathrm{P}}^{2} & =0, C_{\mathrm{S}}=0.25, M=15, \text { and } \\
\varepsilon_{\mathrm{IP}} & =0.25 .
\end{aligned}
$$

Figure 6 presents $\mathrm{OP}_{\mathrm{S}}$ as function of $x_{\mathrm{R}}$ with different positions of the eavesdropper. We can see that the positions of the relays significantly impact on the value of $\mathrm{OP}_{\mathrm{S}}$. Furthermore, optimal values of $x_{\mathrm{R}}$ at which the system obtains the best performance exist. In this figure, it can be seen that the positions of the eavesdropper also affect on the outage performance. Indeed, when the node $\mathrm{E}$ is near the secondary transmitters, the OP value is higher, and vice versa. 


\section{Conclusion}

In this paper, we designed the simple and efficient power allocation strategy for the secondary transmitters to satisfy the primary QoS and to control the IP at the eavesdropper. To overcome the limited transmit power issue, and to mitigate the impact of the primary cochannel interference and hardware imperfection, the bestrelay selection method was used to enhance the outage performance for the secondary network. Moreover, the performance of the considered protocol was evaluated via both simulation and analysis. The obtained results presented that the outage performance can be enhanced by placing the relays at optimal positions, increasing the number of relays, and equipping the legitimate nodes with good transceiver hardware.

\section{Acknowledgements}

This research is funded by Posts and Telecommunications Institute of Technology (PTIT) under grant number 07-HV2019-RD_ĐT

\section{References}

[1] A. D. Wyner (1975) The wire-tap channel. The Bell System Technical Journal, 54(8): 1355-1387.

[2] P. K. Gopala, L. Lai, and H. E. Gamal (2008) On the Secrecy Capacity of Fading Channels. IEEE Transactions on Information Theory, 54(10): 4687-4698.

[3] L. J. Rodriguez, N. H. Tran, T. Q. Duong, T. Le-Ngoc, M. Elkashlan, and S. Shetty (2015) Physical Layer Security in Wireless Cooperative Relay Networks: State of the Art and Beyond. IEEE Communications Magazine, 53(12): 32 39.

[4] I. Krikidis (2010) Opportunistic relay selection for cooperative networks with secrecy constraints. IET Communications, 4(15): 1787-1791.

[5] T. T. Duy, T. Q. Duong, T. L. Thanh, and V. N. Q. Bao (2015) Secrecy Performance Analysis with Relay Selection Methods under Impact of Co-channel Interference. IET Communications, 9(11): 1427-1435.

[6] Tiep M. Hoang, Trung Q. Duong, Himal A. Suraweera, Chintha Tellambura, and H. Vincent Poor (2015) Cooperative Beamforming and User Selection for Improving the Security of Relay-Aided Systems. IEEE Transactions on Communications, 63(12): 5039 - 5051.

[7] I. Krikidis, J. Thompson, and S. Mclaughlin (2009) Relay selection for secure cooperative networks with jamming. IEEE Transactions on Wireless Communications, 8(10): 5003-5011.

[8] Y. Liu, L. Wang, T. T. Duy, M. Elkashlan, Trung Q. Duong (2015) Relay Selection for Security Enhancement in Cognitive Relay Networks. IEEE Wireless Communications Letters, 4(1): 46-49.

[9] T. M. Hoang, T. Q. Duong, N.-S. Vo, and C. Kundu (2017) Physical Layer Security in Cooperative Energy Harvesting Networks with a Friendly Jammer," IEEE Wireless Communications Letters, 6(2): 174-177.

[10] P. T. Tin, and T. T. Duy (2019) Power Allocation Strategies for Dual-hop Relay Protocols with Best Relay Selection under Constraint of Intercept Probability. ICT Express, 5(1): 52-55.
[11] Y. Zou, X. Wang, and W. Shen (2013) Intercept probability analysis of cooperative wireless networks with best relay selection in the presence of eavesdropping attack. In Proc. IEEE ICC2013, Budapest, Hungary, 21832187.

[12] Y. Zou, X. Wang, W. Shen, L. Hanzo (2014) Security versus reliability analysis of opportunistic relaying. IEEE Transactions Vehicular Technology, 63(6): 2653-2661.

[13] Y. Zou (2017) Physical-Layer Security for Spectrum Sharing Systems. IEEE Transactions on Wireless Communications, 16(2): 1319 - 1329.

[14] L. Wang, K. J. Kim, T. Q. Duong, M. Elkashlan, and H. V. Poor (2015) Security Enhancement of Cooperative Single Carrier Systems. IEEE Transactions on Information Forensics Security, 10(1): 90-103.

[15] A. Al-Nahari (2016) Physical layer security using massive multiple-input and multiple-output: passive and active eavesdroppers. IET Communications, 10(1): $50-56$.

[16] W. Wang, K. C. The, S. Luo, and K. H. Li (2017) Secure Transmission in MISOME Wiretap Channels with Half and Full-Duplex Active Eavesdroppers. In Proc. of Globecom2017, Singapore, 1-6.

[17] Q. V. Do, T. N. K. Hoan, and I. Koo (2019) Optimal Power Allocation for Energy-efficient Data Transmission Against Full-duplex Active Eavesdroppers in Wireless Sensor Networks. IEEE Sensors Journal, 1-14, doi: 10.1109/JSEN.2019.2904523

[18] A. Kuhestani, A. Mohammadi, and M. Mohammadi (2018) Joint Relay Selection and Power Allocation in Large-Scale MIMO Systems With Untrusted Relays and Passive Eavesdroppers. IEEE Transactions on Information Forensics and Security, 13(2): $341-355$.

[19] L. Hu, H. Wen, B. Wu, J. Tang, F. Pan, and R.-F. Liao (2018) Cooperative-Jamming-Aided Secrecy Enhancement in Wireless Networks With Passive Eavesdroppers. IEEE Transactions on Vehicular Technology, 67(3): 2108 2117.

[20] E. Bjornson, J. Hoydis, M. Kountouris, and M. Debbah (2013). Hardware impairments in large-scale miso systems: Energy efficiency, estimation, and capacity limits. In Proc. of DSP2013, Santorini, Greece, 1-6.

[21] M. Matthaiou, and A. Papadogiannis (2013). Two-way relaying under the presence of relay transceiver hardware impairments. IEEE Communications Letters, 17(6): 11361139.

[22] T. T. Duy, Trung Q. Duong, D.B. da Costa, V.N.Q. Bao, and M. Elkashlan (2015) Proactive Relay Selection with Joint Impact of Hardware Impairment and Co-channel Interference. IEEE Transactions on Communications, 63(5): 1594-1606.

[23] J. N. Laneman, D. N. Tse, and G. W. Wornell (2004) Cooperative Diversity in Wireless Networks: Efficient Protocols and Outage Behavior. IEEE Transactions on Information Theory, 50(12): 3062 - 3080.

[24] S. Q. Nguyen, H. T. Nguyen, D. D. Van, and W.-J. Hwan (2019) Exact Outage Analysis of Cognitive Energy Harvesting Relaying Networks under Physical Layer Security. EAI Endorsed Transactions on Industrial Networks and Intelligent Systems, 6(18): 1-15.

[25] J. Mo, M. Tao and Y. Liu (2012) Relay Placement for Physical Layer Security: A Secure Connection Perspective. IEEE Communications Letters, 16(6): 878 - 881. 